PROCEEDINGS OF THE

AMERICAN MATHEMATICAL SOCIETY

Volume 134, Number 12, December 2006, Pages 3577-3583

S 0002-9939(06)08744-2

Article electronically published on June 27, 2006

\title{
A VARIATIONAL METHOD IN FIXED POINT RESULTS WITH INWARDNESS CONDITIONS
}

\author{
D. AZÉ AND J.-N. CORVELLEC
}

(Communicated by Jonathan M. Borwein)

\begin{abstract}
We generalize, in a metric space setting, the result due to Lim (2000), that a weakly inward multivalued contraction, defined on a nonempty closed subset of a Banach space, has a fixed point. The simple proof of this generalization, avoiding the use of a transfinite induction as in Lim's paper, is based on Ekeland's variational principle (1974), along the lines of Hamel (1994) and Takahashi (1991). Moreover, we give a sharp estimate for the distance from any point to the fixed point set.
\end{abstract}

\section{InTRODUCTION AND NOTATION}

In this note, we consider a metric space $(X, d)$, a nonempty subset $D$ of $X$, and a multifunction $T: D \rightarrow 2^{X}$ with nonempty values, and we let $\mathcal{F}_{T}:=\{x \in D:$ $x \in T(x)\}$ denote the (possibly empty) set of fixed points of $T$. We shall assume that $T$ has a closed graph $\mathcal{G}_{T}:=\{(x, y) \in D \times X: y \in T(x)\}$. Given $0 \leq \kappa<1$, for $0<\varepsilon<(1-\kappa)(1+\kappa)^{-1}$, we let

$$
\sigma_{\varepsilon}:=\frac{1-\varepsilon}{1+\varepsilon}-\kappa>0,
$$

and we consider the following property, hereafter denoted by $(P)_{\sigma_{\varepsilon}}$ : for every $(x, y) \in \mathcal{G}_{T}$ with $y \neq x$, there exist $u \in X$ and $z \in D$ such that

$$
d(x, y)=d(x, u)+d(u, y), \quad d(u, z)<\varepsilon d(u, x),
$$

and

$$
d(y, T(z)) \leq \kappa d(x, z) .
$$

(Note that since $\varepsilon \leq 1$, both $u$ and $z$ differ from $x$.) We say that $T$ satisfies property $(P)_{1-\kappa}$ if $T$ satisfies property $(P)_{\sigma_{\varepsilon}}$ for all $0<\varepsilon<(1-\kappa)(1+\kappa)^{-1}$.

Let us first compare these properties with more standard notions. Recall that $T$ is a contraction of modulus $\kappa(0 \leq \kappa<1)$ if

$$
e(T(x), T(z)):=\sup _{y \in T(x)} d(y, T(z)) \leq \kappa d(x, z) \quad \text { for all } x, z \in D
$$

(where $\left.d(y, T(z)):=\inf _{w \in T(z)} d(y, w)\right)$. Of course, this is equivalent to

$$
\mathcal{H}(T(x), T(z)) \leq \kappa d(x, z) \quad \text { for all } x, z \in D,
$$

Received by the editors June 24, 2005.

2000 Mathematics Subject Classification. Primary 47H10; Secondary 49J53.

(C)2006 American Mathematical Society 
where $\mathcal{H}(T(x), T(z)):=\max \{e(T(x), T(z)), e(T(z), T(x))\}$ is the classical Hausdorff distance between $T(x)$ and $T(z)$. If $T$ is a contraction of modulus $\kappa$, and if for every $(x, y) \in \mathcal{G}_{T}$ with $y \neq x$ there exists $u \in X$ such that

$$
d(x, y)=d(x, u)+d(u, x) \quad \text { and } \quad d(u, D)<\varepsilon d(u, x),
$$

then (11) and (2) are satisfied, for some $z \in D$. Note further that if $D$ is closed and if $T$ is a contraction with (nonempty) closed values, then $\mathcal{G}_{T}$ is closed.

On the other hand, if $(X,\|\cdot\|)$ is a normed space (and $d(x, y):=\|x-y\|)$, and if for some $\varepsilon>0$ we have

$$
\inf _{t \geq 1, z \in D}\|y-x-t(z-x)\|<\varepsilon\|y-x\| \quad \text { for all }(x, y) \in \mathcal{G}_{T}, y \neq x,
$$

then $T$ satisfies (4) (indeed, if $(x, y) \in \mathcal{G}_{T}$ with $y \neq x$ and if $z \in D$ and $t \geq 1$ are such that $\|y-x-t(z-x)\|<\varepsilon\|y-x\|$, then (44) holds with $u:=x+t^{-1}(y-x)$ since $\|u-z\|<\varepsilon\|u-x\|$ - of course, (4) and (5) are not equivalent, for the set $\{u \in X:\|x-y\|=\|x-u\|+\|u-y\|\}$ may in general differ from the geometrical segment joining $x$ and $y$ ). Now, (5) clearly holds for every $\varepsilon>0$ if and only if

$$
T(x)-x \subset \overline{[1,+\infty[(D-x)} \quad \text { for all } x \in D .
$$

Summing up this discussion, we see that if $D$ is a (nonempty) closed subset of a normed space $X$, and if $T: D \rightarrow 2^{X} \backslash\{\emptyset\}$ is a closed-valued contraction of modulus $\kappa$ verifying ([6), then $T$ satisfies property $(P)_{1-\kappa}$.

Assume now that $D=X$. Given $0 \leq \kappa<1$, we say that the multifunction $T: X \rightarrow 2^{X} \backslash\{\emptyset\}$ defined on the metric space $(X, d)$ is a directional contraction of modulus $\kappa$ if $\mathcal{G}_{T}$ is closed and if for every $(x, y) \in \mathcal{G}_{T}$ with $y \neq x$, there exists $z \in X \backslash\{x\}$ such that

$$
d(x, y)=d(x, z)+d(z, y) \quad \text { and } \quad d(y, T(z)) \leq \kappa d(x, z) .
$$

Observe that a closed-valued contraction is a directional contraction: $\mathcal{G}_{T}$ is closed, while if $(x, y) \in \mathcal{G}_{T}$ with $y \neq x$ and if $T$ satisfies (3), then (7) is satisfied with $z:=y$. Moreover, if $T$ satisfies (77), then $T$ satisfies property $(P)_{\sigma_{\varepsilon}}$ (with $u:=z$ ) for all $0<\varepsilon<(1-\kappa)(1+\kappa)^{-1}$. Thus, if $T: X \rightarrow 2^{X} \backslash\{\emptyset\}$ is a directional contraction of modulus $\kappa$, then $T$ satisfies property $(P)_{1-\kappa}$.

In 7, Lim proved the following nice result: if $D$ is a closed subset of a Banach space $X$ and $T: D \rightarrow 2^{X} \backslash\{\emptyset\}$ is a closed-valued contraction satisfying (6) - that is to say, $T$ is a weakly inward contraction - then $T$ has a fixed point. Here, we give an elementary proof of a generalization of this result, using property $(P)_{\sigma_{\varepsilon}}$ and a variational method directly derived from the Ekeland variational principle, thus avoiding the use of transfinite induction as in the quoted reference. Moreover, we also provide an estimate for the distance $d\left(x, \mathcal{F}_{T}\right)$. The use of variational methods in fixed point theory dates back to the celebrated paper of Caristi 3 . However, our point of view is quite different, more in line with the method of Hamel [6] and Takahashi 12, and is based upon an immediate application of the basic Ekeland's principle in the spirit of Penot [10, 11].

Our main result also provides an extension of a result in Clarke [4, dealing with continuous single-valued directional contractions, as well as containing the classical result of Nadler 9 .

In the sequel, we shall use the following notation: given a function $f: X \rightarrow$ $\mathbb{R} \cup\{+\infty\}$, if $-\infty<\alpha<\beta \leq+\infty$, we set

$$
[f \leq \beta]:=\{x \in X: f(x) \leq \beta\}
$$


(so that $[f \leq+\infty]=X$ ), and we define in a similar way the sets $[f<\beta],[f>\alpha]$, and $[\alpha<f<\beta]:=[f<\beta] \backslash[f \leq \alpha]$. We say that $f$ is proper if $\operatorname{dom} f:=[f<+\infty] \neq \emptyset$.

\section{FiXed POINT RESULtS}

Let $X$ be a metric space endowed with the metric $d$, and let $f: X \rightarrow \mathbb{R} \cup\{+\infty\}$ be a function. A point $x \in X$ is said to be a d-point of $f$ if

$$
f(x)<f(z)+d(z, x) \quad \text { for all } z \in X, z \neq x .
$$

(Observe that $d$-points are in $\operatorname{dom} f$, and that global minima are $d$-points.) With our notation, the basic form of Ekeland's variational principle is the following (see [11, Theorem B]; see also [2, Theorem 2.1]):

Theorem 2.1. If $(X, d)$ is complete and $f: X \rightarrow \mathbb{R} \cup\{+\infty\}$ is proper, lower semicontinuous, and bounded from below, then $f$ has a d-point.

This result is proved through a simple iterative procedure involving a sequence of sets of the type $M_{x}:=\{z \in X: f(z)+d(z, x) \leq f(x)\} \subset[f \leq f(x)], x \in X$. It is readily seen that $x$ is a $d$-point of $f$ if and only if $M_{x}=\{x\}$, and that, using the triangle inequality we have

$\bar{x} \in M_{x}$ is a $d$-point of the restriction of $f$ to $M_{x} \Longrightarrow \bar{x}$ is a $d$-point of $f$.

Thus, applying Theorem 2.1 to the restriction of $f$ to $M_{x}$, we immediately obtain the following.

Corollary 2.1. Let $(X, d)$ be a metric space and let $f: X \rightarrow \mathbb{R} \cup\{+\infty\}$ be proper, lower semicontinuous, and bounded from below. Assume that for every $x \in \operatorname{dom} f$, the set $[f \leq f(x)]$ is complete. Then, for every $x \in X, f$ has a $d$-point in $M_{x}$.

Note that Corollary 2.1 applies to $([f<\beta], d), \beta \in \mathbb{R} \cup\{+\infty\}$, whenever $(X, d)$ is complete. The result with $(X, d)$ complete is given in [11, Theorem B]. We finally state the following immediate consequence of Corollary 2.1 which we shall use for the proof of our main result.

Corollary 2.2. Let $(X, d)$ be a metric space and let $f: X \rightarrow \mathbb{R} \cup\{+\infty\}$ be a lower semicontinuous function. Assume that for every $x \in \operatorname{dom} f$ the set $[f \leq f(x)]$ is complete, and that $\mu:=\inf _{X} f \in \mathbb{R}$. Set $S:=[f \leq \mu]$. Then, the following are equivalent:

(a) $f$ has no d-point in $X \backslash S$;

(b) for every $x \in X$, there exists $\bar{x} \in M_{x} \cap S$ (i.e., $f(x)-\mu \geq d(x, \bar{x})$ ).

(Indeed, a $d$-point of $f$ in $M_{x}$, given by Corollary 2.1. must be in $S$ if (a) holds, and conversely, $M_{x} \neq\{x\}$ for $x \in X \backslash S$ if (b) holds.) Corollary 2.2 slightly extends [6. Theorem 2], which was itself an extension of the results of [12].

Remark 2.1. Let $(X, d)$ be complete, let $f: X \rightarrow \mathbb{R} \cup\{+\infty\}$ be lower semicontinuous, and let $-\infty<\alpha<\beta \leq+\infty$. Then, applying Corollary 2.2 to the metric space $([f<\beta], d)$ and to the function $g:[f<\beta] \rightarrow \mathbb{R} \cup\{+\infty\}$ defined by $g(x):=(f(x)-\alpha)^{+}$, yields that the following are equivalent:

(a) for every $x \in[\alpha<f<\beta]$ there exists $y \in[f<\beta] \backslash\{x\}$ such that $f(x)-\alpha \geq$ $(f(y)-\alpha)^{+}+d(y, x)$

(b) for every $x \in[\alpha<f<\beta]$ there exists $\bar{x} \in[f \leq \alpha]$ such that $f(x)-\alpha \geq d(x, \bar{x})$.

See [2] and the references therein for related results. 
The following is the main result of this note.

Theorem 2.2. Let $D$ be a nonempty subset of a complete metric space $(X, d)$, and let $T: D \rightarrow 2^{X}$ be a multifunction with nonempty values and closed graph. Let $0 \leq \kappa<1$, let $\varepsilon>0$ be such that

$$
\sigma_{\varepsilon}:=\frac{1-\varepsilon}{1+\varepsilon}-\kappa>0,
$$

and assume that $T$ satisfies property $(P)_{\sigma_{\varepsilon}}$ (recall (1)-(2) ). Then, $\mathcal{F}_{T} \neq \emptyset$ and

$$
d(x, T(x)) \geq \sigma_{\varepsilon} d\left(x, \mathcal{F}_{T}\right) \quad \text { for all } x \in D .
$$

Proof. Assume first that $\kappa>0$. Let $(x, y) \in \mathcal{G}_{T}, y \neq x$, be fixed. According to property $(P)_{\sigma_{\varepsilon}}$, we find $u \in X$ and $z \in D$ such that $d(x, y)=d(x, u)+d(u, y)$, $d(u, z)<\varepsilon d(u, x)$, and $d(y, T(z)) \leq \kappa d(x, z)$. Then

$$
d(x, z) \leq d(x, u)+d(u, z)<(1+\varepsilon) d(x, u)
$$

and

$$
d(y, T(z)) \leq \kappa d(x, z)<\kappa(1+\varepsilon) d(x, u),
$$

so that we find $w \in T(z)$ with

$$
d(y, w) \leq \kappa(1+\varepsilon) d(x, u) .
$$

On the other hand, we also have

$$
\begin{aligned}
d(z, w) & \leq d(z, u)+d(u, y)+d(y, w) \\
& =d(z, u)+d(x, y)-d(x, u)+d(y, w) \\
& <\varepsilon d(u, x)+d(x, y)-d(x, u)+\kappa(1+\varepsilon) d(x, u)
\end{aligned}
$$

so that

$$
d(x, y)>d(z, w)+(1-\varepsilon-\kappa(1+\varepsilon)) d(x, u)=d(z, w)+\sigma_{\varepsilon}(1+\varepsilon) d(x, u) .
$$

Now, consider $X \times X$ as endowed with the metric

$$
\tilde{d}\left(\left(x^{\prime}, y^{\prime}\right),\left(x^{\prime \prime}, y^{\prime \prime}\right)\right):=\sigma_{\varepsilon} \max \left\{d\left(x^{\prime}, x^{\prime \prime}\right), \frac{1}{\kappa} d\left(y^{\prime}, y^{\prime \prime}\right)\right\} .
$$

Since $\mathcal{G}_{T}$ is closed in $(X \times X, \tilde{d}),\left(\mathcal{G}_{T}, \tilde{d}\right)$ is complete. Consider the continuous, nonnegative function $f:=d_{\mid \mathcal{G}_{T}}$ (the restriction of $d$ to $\mathcal{G}_{T}$ ). Then, combining (8), (9), and (10), we see that for every $(x, y) \in \mathcal{G}_{T}$ with $y \neq x$, we find $(z, w) \in \mathcal{G}_{T}$ such that

$$
f(x, y)>f(z, w)+\tilde{d}((x, y),(z, w)) .
$$

Thus, $f$ has no $\tilde{d}$-point in $[f>0]$, and we deduce from Corollary 2.2 that inf $f=0$, that $[f \leq 0]=\left\{(x, x): x \in \mathcal{F}_{T}\right\} \neq \emptyset$, and that for every $(x, y) \in \mathcal{G}_{T}$ there exists $\bar{x} \in \mathcal{F}_{T}$ such that

$$
d(x, y) \geq \tilde{d}((x, y),(\bar{x}, \bar{x})) \geq \sigma_{\varepsilon} d(x, \bar{x}) \geq \sigma_{\varepsilon} d\left(x, \mathcal{F}_{T}\right),
$$

so that $d(x, T(x)) \geq \sigma d\left(x, \mathcal{F}_{T}\right)$, since $y$ is arbitrary in $T(x)$.

If $\kappa=0$, considering $\tilde{\kappa}>0$ such that $\tilde{\sigma}_{\varepsilon}:=(1-\varepsilon)(1+\varepsilon)^{-1}-\tilde{\kappa}>0$, we see that $T$ satisfies property $(P)_{\tilde{\sigma}_{\varepsilon}}$, so that, arguing as above and then letting $\tilde{\kappa} \rightarrow 0$ yields the conclusion. 
Corollary 2.3. Let $D$ be a nonempty subset of a complete metric space $(X, d)$, let $T: D \rightarrow 2^{X}$ be a multifunction with nonempty values and closed graph, and let $0 \leq \kappa<1$. Assume that $T$ satisfies property $(P)_{1-\kappa}$. Then, $\mathcal{F}_{T} \neq \emptyset$ and $d(x, T(x)) \geq(1-\kappa) d\left(x, \mathcal{F}_{T}\right)$ for all $x \in D$.

Proof. Recall that $T$ satisfying property $(P)_{1-\kappa}$ means that it satisfies property $(P)_{\sigma_{\varepsilon}}$ for any $0<\varepsilon<(1-\kappa)(1+\kappa)^{-1}$. Thus, the conclusion follows from Theorem 2.2 .

Remark 2.2. Taking into account what we already said in the introduction, Corollary 2.3 is an extension of Lim's result [7, Theorem 1], where $D$ is a nonempty closed subset of a Banach space $X$, and $T$ is a contraction with nonempty, closed values satisfying the inwardness condition (6). This result was previously established for single-valued maps by Martinez-Yanez [8], and by Xu [13, Theorem 3.3] under the additional assumption that each $x \in D$ has a nearest point in $T(x)$. Corollary 2.3 also extends Clarke's Theorem 7.6.2 in [4, where $T$ is a continuous single-valued, directional contraction from the complete metric space $X$ into itself, as well as it contains Nadler's theorem: every multi-valued contraction with nonempty, closed values, from a complete metric space into itself, has a fixed point (indeed, Nadler considers multifunctions with bounded values - see Remark 2.3 below). A simple example of a (single-valued) directional contraction which is not a contraction is given in [4, Remark 7.6.3].

We note that a variational proof of Nadler's theorem was already given by Takahashi [12, while Clarke's proof of his result also relies on Ekeland's principle, applied to the function $f(x):=d(x, T(x))$, which is continuous if so is (the single-valued map) $T$. Our proof of Theorem 2.2 suggests that it is more appropriate to work in $\mathcal{G}_{T}$ - that is, in the product space $X \times X$ - when possible (in the same spirit, see [1, Section 5]). Still, we now give a variant of Theorem [2.2, where we apply Corollary 2.2 to the function $x \mapsto d(x, T(x))$; see Remark 2.3 below for comments.

Theorem 2.3. Let $D$ be a nonempty closed subset of a complete metric space $(X, d)$, and let $T: D \rightarrow 2^{X}$ be a multifunction with nonempty, closed values. Assume that every $x \in D$ has a nearest point in $T(x)$, and that the function $x \mapsto$ $d(x, T(x))$ is lower semicontinuous on $D$. Let further $0 \leq \kappa<1$ and $\varepsilon>0$ be such that

$$
\sigma:=\frac{1-\varepsilon}{1+\varepsilon}-\kappa>0,
$$

and assume that for every $(x, y) \in \mathcal{G}_{T}$ with $y \neq x$, there exist $u \in X$ and $z \in D$ such that

$$
d(x, y)=d(x, u)+d(u, y), \quad d(u, z)<\varepsilon d(u, x),
$$

and

$$
d(z, T(z)) \leq d(z, y)+\kappa d(z, x) .
$$

Then, $\mathcal{F}_{T} \neq \emptyset$ and

$$
d(x, T(x)) \geq \sigma d\left(x, \mathcal{F}_{T}\right) \quad \text { for all } x \in D .
$$

Proof. We consider $D$ as endowed with the metric $\tilde{d}(x, y):=\sigma d(x, y)$ and the lower semicontinuous function $f: D \rightarrow \mathbb{R}_{+}$defined by $f(x):=d(x, T(x))$. Then, $(D, \tilde{d})$ is complete and $[f \leq 0]=\mathcal{F}_{T}$. Let $x \in D$ be such that $f(x)>0$, and let $y \in T(x)$ 
be such that $f(x)=d(x, y)$ - so that $y \neq x$. Then let $u \in X$ and $z \in D$ be as in (11) and (12). We obtain from (11) that $d(z, x)<(1+\varepsilon) d(u, x)$ and

$$
\begin{aligned}
d(z, y)+\frac{1-\varepsilon}{1+\varepsilon} d(z, x) & <d(z, y)+(1-\varepsilon) d(u, x) \\
& =d(z, y)+d(x, y)-d(u, y)-\varepsilon d(u, x) \\
& <d(z, y)-(d(u, y)+d(u, z))+d(x, y) \leq d(x, y) .
\end{aligned}
$$

Then, using also (12), we have

$$
d(z, T(z))+\sigma d(z, x) \leq d(z, y)+\frac{1-\varepsilon}{1+\varepsilon} d(z, x),
$$

so that, combining the previous inequalities, we have $f(z)+\tilde{d}(z, x)<f(x)$. Thus, $f$ has no $\tilde{d}$-point in $[f>0]$, and we deduce from Corollary 2.2 that $[f \leq 0]=\mathcal{F}_{T} \neq \emptyset$, and that for every $x \in D$ there exists $\bar{x} \in \mathcal{F}_{T}$ such that $d(x, T(x)) \geq \sigma d(x, \bar{x}) \geq$ $\sigma d\left(x, \mathcal{F}_{T}\right)$.

Remark 2.3. The assumption that $x \mapsto d(x, T(x))$ is lower semicontinuous, in Theorem [2.3, is satisfied, for example, if $T$ is $\mathcal{H}$-upper semicontinuous (" $\mathcal{H}$ " is for Hausdorff), that is, if $\lim _{z \rightarrow x} e(T(z), T(x))=0$ for all $x \in D$.

Note that (2) implies (12) since $d(z, T(z)) \leq d(z, y)+d(y, T(z))$. Similarly, if $T$ is a contraction of modulus $\kappa$, then $T$ satisfies

$$
d(z, T(z)) \leq d(z, T(x))+\kappa d(z, x) \quad \text { for all } z, x \in D .
$$

When the multifunction $T$ has unbounded values, the requirement that it be a contraction is difficult to satisfy, in general. We show in the following simple example that condition (13) is strictly weaker than the contractiveness of $T$.

Example 2.1. Let $X:=\mathbb{R}, D:=[0,1]$, and $T: D \rightarrow 2^{X}$ be defined by

$$
T(x):= \begin{cases}\left(-\infty, \frac{x}{2}+\frac{1}{4}\right] & \text { if } x>0, \\ {\left[0, \frac{1}{4}\right]} & \text { if } x=0 .\end{cases}
$$

Then, for any $x, z \in[0,1]$ we have $d(z, T(x))=\left(z-\frac{x}{2}-\frac{1}{4}\right)^{+}$, whence an easy computation yields $d(z, T(z))-d(z, T(x)) \leq \frac{1}{2}|z-x|$, so that (13) is verified with $\kappa:=\frac{1}{2}$. But $T$ is not a contraction since $\mathcal{H}(T(0), T(x))=+\infty$ for any $x>0$. Observe that $T$ indeed satisfies property $(P)_{1-\kappa}$, so that Theorem 2.2 applies, as well as Theorem 2.3 . Since $\mathcal{F}_{T}=\left[0, \frac{1}{2}\right]$, we see in this example that the estimate $d(x, T(x)) \geq(1-\kappa) d\left(x, \mathcal{F}_{T}\right)$ is exact.

\section{ACKNOWLEDGEMENT}

We thank the referee for drawing our attention to Clarke's result, which led us to greatly improve the presentation of the paper.

\section{REFERENCES}

[1] D. AzÉ And J.-N. CoRvellec, Characterizations of error bounds for lower semicontinuous functions on metric spaces, ESAIM Control, Optim. Calc. Var. 10 (2004), 409-425. MR2084330 (2005e:49027)

[2] D. AzÉ And J.-N. CoRvellec, Variational methods in classical open mapping theorems, J. Convex Anal. 13 (2006), in press.

[3] J. CARISTI, Fixed point theorems for mapping satisfying inwardness conditions, Trans. Amer. Math. Soc. 215 (1976), 241-251. MR0394329 (52:15132) 
[4] F. H. Clarke, Optimization and Nonsmooth Analysis, Classics in Applied Mathematics, Vol. 5, SIAM, Philadelphia, PA, 1990 (originally published by Wiley-Interscience, New York, 1983). MR0709590 (85m:49002)

[5] I. Ekeland, On the variational principle, J. Math. Anal. Appl. 47 (1974), 324-353. MR0346619 (49:11344)

[6] A. Hamel, Remarks to an equivalent formulation of Ekeland's variational principle, Optimization 31 (1994), 233-238. MR1307103 (95h:49019)

[7] T. C. Lim, A fixed point theorem for weakly inward multivalued contractions, J. Math. Anal. Appl. 243 (2000), 323-327. MR1766943(2001d:47088)

[8] C. Martínez-Yañez, A remark on weakly inward contractions, Nonlinear Anal. 16 (1991), 847-848. MR1106372 (92g:47086)

[9] S. B. NadleR JR., Multi-valued contraction mappings, Pacific J. Math. 30 (1969), 475-488. MR0254828 (40:8035)

[10] J.-P. Penot, A short constructive proof of Caristi's fixed point theorem, Publ. Math. Univ. Pau (1976), 1-3.

[11] J.-P. PEnot, The drop theorem, the petal theorem and Ekeland's variational principle, Nonlinear Anal. 10 (1986), 813-822. MR0856865 (87j:49031)

[12] W. TAKAHASHI, Existence theorems generalizing fixed point theorems for multivalued mappings, Fixed Point Theory and Applications (Marseille 1989), 397-406, Pitman Res. Notes Math. Ser., Vol. 252, Longman, Harlow, 1991. MR1122847 (92m:54078)

[13] H. K. Xu, Multivalued nonexpansive mappings in Banach spaces, Nonlinear Anal. 43 (2001), 693-706. MR1808203 (2002b:47122)

UMR CNRS MiP, Université Paul Sabatier, 118 route de Narbonne, 31062 Toulouse CEDEx 4, France

E-mail address: aze@mip.ups-tlse.fr

Laboratoire Mano, Université de Perpignan Via Domitia, 52 avenue Paul Alduy, 66860 Perpignan cedex, France

E-mail address: corvellec@univ-perp.fr 